University of Wollongong

Research Online

Faculty of Engineering and Information

Faculty of Engineering and Information

Sciences - Papers: Part A

Sciences

$1-1-2013$

\title{
A novel cost effective method for vehicle tire-road friction coefficient estimation
}

Boyuan $\mathrm{Li}$

University of Wollongong, bl995@uowmail.edu.au

Haiping Du

University of Wollongong, hdu@uow.edu.au

Weihua Li

University of Wollongong, weihuali@uow.edu.au

Follow this and additional works at: https://ro.uow.edu.au/eispapers

Part of the Engineering Commons, and the Science and Technology Studies Commons

Research Online is the open access institutional repository for the University of Wollongong. For further information contact the UOW Library: research-pubs@uow.edu.au 


\title{
A novel cost effective method for vehicle tire-road friction coefficient estimation
}

\begin{abstract}
The tire-road friction coefficient is critical to vehicle longitudinal, lateral and roll dynamics and control because tire is the only contact part between the vehicle body and the road. However, direct measurement of tire-road friction coefficient is impossible in practice. This paper presents a novel cost effective method for vehicle tire-road friction coefficient estimation. This method only needs the measurements of the wheel angular velocity, the traction/brake torque and the longitudinal acceleration, which are all available from the commonly installed sensors in ordinary passenger vehicles, and can be used to estimate the individual tire-road friction coefficient. There are three steps in the proposed method. Firstly, the longitudinal slip ratio is estimated by using a nonlinear filter with the measured wheel angular velocity. Then the tire longitudinal force is estimated by using a Kalman filter with the measured traction/ brake torque and longitudinal acceleration. At last, the friction coefficient is estimated by using the recursive least squares (RLS) method and the results obtained from the first two steps. Numerical simulations are provided to validate the effectiveness of the proposed method. It is shown by the simulation results that the proposed method is effective in estimating the tire-road friction coefficient.
\end{abstract}

\section{Keywords}

coefficient, friction, vehicle, method, road, estimation, effective, cost, tire, novel

Disciplines

Engineering | Science and Technology Studies

\section{Publication Details}

B. Li, H. Du \& W. Li, "A novel cost effective method for vehicle tire-road friction coefficient estimation," in 2013 IEEE/ASME International Conference on Advanced Intelligent Mechatronics (AIM), 2013, pp. 1528-1533. 


\title{
A Novel Cost Effective Method for Vehicle Tire-Road Friction Coefficient Estimation
}

\author{
B. Li, H. Du, and W. Li
}

\begin{abstract}
The tire-road friction coefficient is critical to vehicle longitudinal, lateral and roll dynamics and control because tire is the only contact part between the vehicle body and the road. However, direct measurement of tire-road friction coefficient is impossible in practice. This paper presents a novel cost effective method for vehicle tire-road friction coefficient estimation. This method only needs the measurements of the wheel angular velocity, the traction/brake torque and the longitudinal acceleration, which are all available from the commonly installed sensors in ordinary passenger vehicles, and can be used to estimate the individual tire-road friction coefficient. There are three steps in the proposed method. Firstly, the longitudinal slip ratio is estimated by using a nonlinear filter with the measured wheel angular velocity. Then the tire longitudinal force is estimated by using a Kalman filter with the measured traction/brake torque and longitudinal acceleration. At last, the friction coefficient is estimated by using the recursive least squares (RLS) method and the results obtained from the first two steps. Numerical simulations are provided to validate the effectiveness of the proposed method. It is shown by the simulation results that the proposed method is effective in estimating the tire-road friction coefficient.
\end{abstract}

Key words - tire-road friction, friction coefficient estimation, velocity estimation, tire force estimation

\section{INTRODUCTION}

Because the tire is the only contact part between the vehicle body and the road, the tire-road friction coefficient is critical to vehicle longitudinal, lateral and roll dynamics and control. For example, for the electronic stability control (ESC) system, the yaw rate will be controlled to prevent skidding. If the friction coefficient can be estimated, the yaw rate feedback with the knowledge of friction coefficient can be sent back to the controller to control the yaw rate adaptively [1]-[3]. For the four-wheel-driving (4WD) vehicle, the estimation of individual tire friction coefficient can help to detect which wheel needs the maximum drive torque [4]. In the adaptive cruise control and collisionavoidance system, the estimated friction coefficient can help to adjust the braking distance in real time [5]. And for road maintenance in winter, the estimation of friction coefficient

B. $\mathrm{Li}$ is with the School of Electrical, Computer and Telecommunications Engineering, University of Wollongong, Wollongong, NSW 2522, Australia (e-mail: bl995@ uowmail.edu.au).

H. Du is with the School of Electrical, Computer and Telecommunications Engineering, University of Wollongong, Wollongong, NSW 2522, Australia (61-2-42214610 e-mail: hdu@uow.edu.au).

W. Li is with the School of Mechanical, Materials and Mechatronic Engineering, University of Wollongong, Wollongong, NSW 2522, Australia (e-mail: Weihuali@uow.edu.au). can be used to adjust the de-icing materials on the icy road [6].

Many researches have been done on the estimation of tire-road friction coefficient in recent years. Some of them only focus on the vehicle longitudinal motion with vehicle traction or braking operation, and some of them also consider the vehicle lateral motion. Three main methods have been proposed so far. They are slip slop method, extended Kalman filter (EKF) method, and individual wheel friction estimation method.

The slip slope method is a longitudinal friction coefficient estimation method and a lot of papers have studied on this method. Gustafsson [7] developed a slip slope friction estimation system in the traction condition. Müller and Uchanski [8] proposed the slip slope friction estimation in the brake situation. Wang, Alexander and Rajamani [9] developed the slip slope estimation method for the front wheel, rear wheel and four wheel traction/brake systems under linear and nonlinear tire region.

The EKF method is based on both the longitudinal and lateral dynamics. This estimation method includes two parts: the state estimation part, which is done by the EKF, and the friction identification part. Ray [10] developed the EKF method based on a 8 degrees of freedom (DOFs) vehicle dynamics model. Dakhlallah et al. [11] proposed an EKF estimation method based on a simpler vehicle dynamics model, which only considers vehicle longitudinal dynamics, lateral dynamics and yaw dynamics.

The individual wheel friction estimation method [12] is also a vehicle longitudinal dynamics based method. There are three steps in this estimation method: (1) estimate the longitudinal tire force; (2) estimate the tire longitudinal slip ratio; and (3) calculate the friction coefficient by using the recursive least squares (RLS) method. Similar to the slip slope method, the friction coefficient is calculated by the RLS method when the longitudinal slip ratio and longitudinal tire force are estimated.

The slip slope method needs the global positioning system (GPS) to measure the vehicle absolute velocity and the anti-lock braking system (ABS) to measure the wheel angular velocity. The longitudinal tire force can be estimated by the accelerometer, which is not accurate enough. The individual tire force estimation method does not need GPS to measure the absolute vehicle velocity and can estimate the friction coefficient of each tire. However, the estimation results are not accurate and the estimation gain needs to be 
adjusted. The EKF method in the literature needs to measure the tire force, which is quite hard to measure and is not cost effective.

In this paper, a more reliable and cost effective friction estimation method will be developed. This estimator can estimate the friction coefficient individually and is cost effective in terms of the required measurements. The paper is organised as follows. Section 2 presents the basic modeling of the vehicle. Section 3 describes the novel cost effective friction estimation method. Section 4 provides the actual simulation results to validate the effectiveness of the proposed method. Conclusions will be given in Section 5 .

\section{VEHICLE MODELING}

\section{A. Vehicle Dynamics Model}

In this study, the vehicle dynamics model considers the longitudinal dynamics, the lateral dynamics, the yaw dynamics, and the wheel rotation dynamics. The motions of equations are described as

$$
\left[\begin{array}{c}
\dot{v}_{x} \\
\dot{v}_{y} \\
\dot{r} \\
\dot{\omega}_{f} \\
\dot{\omega}_{r}
\end{array}\right]=\left[\begin{array}{c}
\frac{1}{m}\left(F_{x f} \cos \delta_{f}+F_{y f} \sin \delta_{f}+F_{x r}\right)+v_{y} r \\
\frac{1}{m}\left(F_{y f} \cos \delta_{f}-F_{x f} \sin \delta_{f}+F_{y r}\right)-v_{x} r \\
\frac{1}{I}\left(L_{f}\left(F_{y f} \cos \delta_{f}-F_{x f} \sin \delta_{f}\right)-L_{r} F_{y r}\right) \\
\frac{1}{I_{\omega}}\left(-F_{x f} R_{\omega}+T_{f}\right) \\
\frac{1}{I_{\omega}}\left(-F_{x r} R_{\omega}+T_{r}\right)
\end{array}\right]
$$

where $v_{x}, v_{y}, r, \omega_{f}, \omega_{r} \quad$ are longitudinal velocity, lateral velocity, yaw rate, front wheel angular velocity and rear wheel angular velocity, respectively.

$F_{x f}, F_{x r}, F_{y f}, F_{y r}$ are the front and rear longitudinal and lateral forces. $\delta_{f}, T_{f}, T_{r}$ represent the front steer angle and the applied front and rear traction/brake torque, respectively.

\section{B. The Tire Model}

The Dugoff tire model is used in this research, which is presented by the following equations:

$$
\begin{gathered}
\lambda=\frac{\mu F_{z i}\left[1-\varepsilon_{r} u_{i} \sqrt{S_{i}^{2}+\tan ^{2} \alpha_{i}}\right]\left(1-S_{i}\right)}{2 \sqrt{C_{i}^{2} S_{i}^{2}+C_{\alpha}^{2} \tan ^{2} \alpha_{i}}} \\
f(\lambda)=\left\{\begin{array}{cc}
\lambda(2-\lambda) & \text { if } \lambda<1 \\
1 & \text { if } \quad \lambda>1
\end{array}\right. \\
F_{s i}=\frac{C_{\alpha} \tan \alpha_{i}}{1-S_{i}} f(\lambda) \\
F_{t i}=\frac{C_{i} S_{i}}{1-S_{i}} f(\lambda)
\end{gathered}
$$

where $\mu$ is the friction coefficient. $F_{z}, F_{s i}, F_{t i}$ are the vertical load, the side tire force and the tractive tire force, where $\mathrm{i}=\mathrm{f}, \mathrm{r}$ denote front and rear, respectively. $C_{i}$ is the longitudinal cornering stiffness and $C_{\alpha}$ is the lateral cornering stiffness. $S_{i}$ is the longitudinal slip ratio and $\alpha_{i}$ is the lateral slip angle. $u_{i}$ is the vehicle velocity components in wheel plane and $\varepsilon_{r}$ is the constant value.

The integrated vehicle dynamics model and tire model will be used to simulate the actual vehicle motion and validate the proposed method.

\section{The Novel Cost Effective TIRE-RoAd Friction COEFFICIENT ESTIMATION METHOD}

A novel cost effective tire-road friction coefficient estimation method is proposed here. This method only needs to measure the wheel angular velocity (measured by the ABS system), the traction/brake torque and the longitudinal acceleration. In the past years, the measurement of traction torque relies on the engine torque measurement and the measurement of brake torque depends on brake pressure sensor, which is too expensive to be implemented [9]. However, recently the information of engine torque and brake torque can be obtained from CAN bus of the vehicle [12]. The longitudinal acceleration can be measured by the longitudinal accelerometer. The vehicle absolute velocity can be estimated by the nonlinear filter without using the expensive GPS.

There are mainly three steps in this friction estimator: (1) the estimation of the longitudinal slip ratio, which needs to measure the wheel angular velocity; (2) the estimation of the tire longitudinal force, which needs to measure the traction/brake torque and longitudinal acceleration; and (3) the estimation of friction coefficient according to the results of the first two steps. Figure 1 shows the block diagram of the whole procedure.

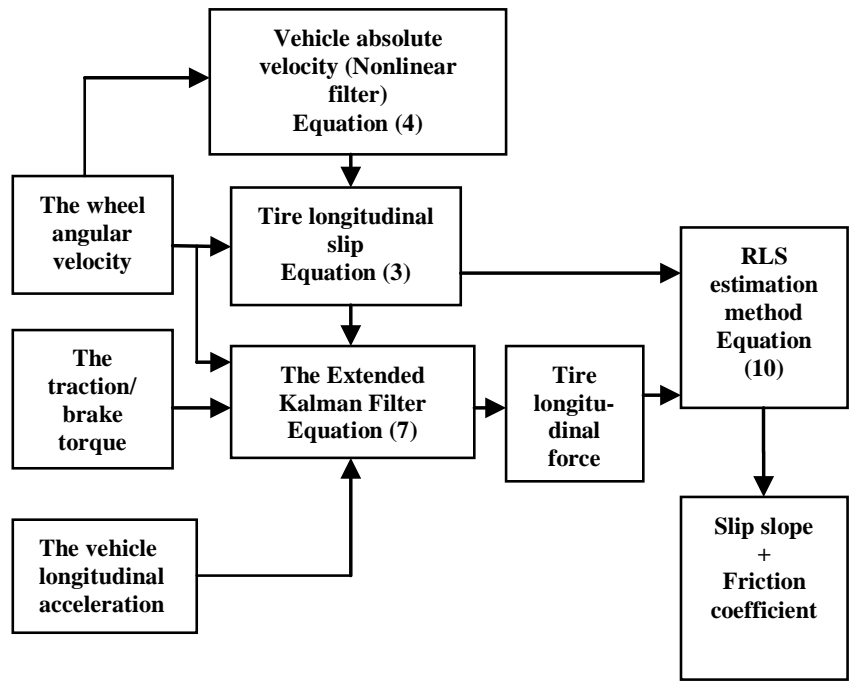

Figure 1. The block diagram of the whole friction estimation procedure 


\section{A. The Estimation of the Vehicle Longitudinal Slip Ratio}

The vehicle longitudinal slip ratio can be calculated by the following equation:

$$
S_{i}=\frac{\omega R_{\omega}-V_{x}}{\max \left(V_{x}, \omega R_{\omega}\right)}
$$

where $\omega$ is the wheel angular velocity, which can be easily measured. $R_{\omega}$ is the effective turn radius.

The vehicle absolute velocity can be estimated by the following nonlinear filter [13]. The mathematical equation of this nonlinear filter is:

$$
\begin{aligned}
& \dot{y}(t)=-R_{g} \cdot \operatorname{Sat}(y(t)-x(t)) \\
& y(t=0)=y_{0}
\end{aligned}
$$

where $y(t)$ is the estimated vehicle absolute velocity. $x(t)$ is the vehicle wheel angular velocity multiplied by wheel radius. ' $R_{g}$ ' denotes the change of vehicle velocity (acceleration), which has the following relationship with traction/brake torque:

$$
R_{g}=\frac{1}{466.5} T_{f, r}
$$

The saturation function $\operatorname{Sat}(x)$ is defined as:

$$
\operatorname{Sat}(x)=\left\{\begin{array}{cc}
1, & x>d \\
-1, & x<-d \\
x / d, & \text { else }
\end{array}\right.
$$

where $d$ is a small number like 0.01 . This velocity estimation method uses the input traction/brake torque to estimate the velocity, which does not need to integrate the measurement value of the acceleration. The measurement value of acceleration has noise and the integration of the value will induce even larger noise, which is not accurate for the estimation of the velocity.

The accuracy of the estimated vehicle absolute velocity is important to the estimation results of vehicle longitudinal slip. Therefore, the simple controller is used to adjust the estimation results of the velocity according to the measured acceleration, which is shown in Figure. 2.

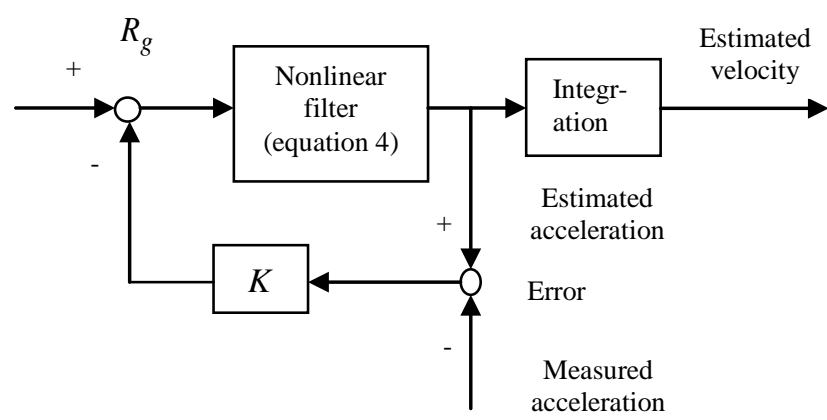

Figure 2. The control block diagram of the absolute velocity estimator

\section{B. The Estimation of the Vehicle Longitudinal Force}

In this method, the vehicle tire force will be estimated instead of using expensive sensors to measure. The vehicle acceleration and the wheel angular velocity can be utilised as the measurement information to update the error gain and improve the estimation results. Therefore, this estimation method is cost effective and reliable. In addition, this estimator can estimate the front and rear tire force individually.

The estimator is written in the discrete time as:

$$
\begin{aligned}
& a_{x}(k+1)=\frac{1}{m}\left(F_{x f}(k)+F_{x r}(k)\right) \\
& a_{\omega f}(k+1)=\frac{1}{I_{\omega}}\left(-F_{x f}(k) R_{\omega}+T_{f}(k)\right) \\
& a_{\omega r}(k+1)=\frac{1}{I_{\omega}}\left(-F_{x r}(k) R_{\omega}+T_{r}(k)\right) \\
& F_{x f}(k+1)=C_{s} S_{f}(k) \\
& F_{x r}(k+1)=C_{s} S_{r}(k)
\end{aligned}
$$

where $a_{\omega f}, a_{\omega r}$ represent the derivative of the wheel angular velocity of front and rear wheel. Equation (7) is further written in the following form as:

$$
\begin{aligned}
& x_{k+1}=f\left(x_{k}, u_{k}, w_{k}\right) \\
& z_{k+1}=h\left(x_{k}, v_{k}\right)
\end{aligned}
$$

where $x_{k}$ is the state vector, $u_{k}$ is the input vector and $z_{k}$ is the measurement value. $w_{k}$ is the processing noise and $v_{k}$ is the measurement noise.

$$
\begin{gathered}
x_{k}=\left[\begin{array}{lllll}
a_{x}(k) & a_{\omega f}(k) & a_{\omega r}(k) & F_{x f}(k) & F_{x r}(k)
\end{array}\right]^{T} \\
u_{k}=\left[\begin{array}{llll}
T_{f}(k) & T_{r}(k) & s_{f}(k) & s_{r}(k)
\end{array}\right] \\
z_{k}=a_{x}(k)
\end{gathered}
$$

After choosing the initial value for $x_{k}{ }^{-}$and $P_{k}{ }^{-}$, the Extended Kalman Filter (EKF) recursive algorithm can be achieved (see Figure 3) [14]. The left column is the time update process and the state vector can be updated by equation (7) in discrete time. The right column is the measurement update process, which means that the state vector also needs to be updated according to the error between the measured value and estimation value. 


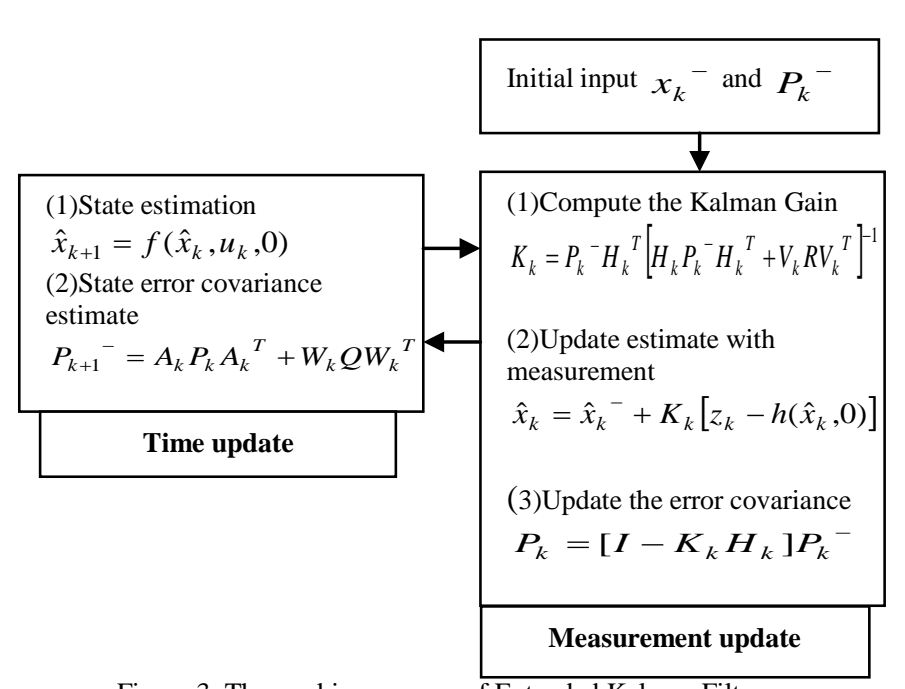

Figure 3. The working process of Extended Kalman Filter

$A_{k}$ is the Jacobian matrix of partial derivative of $f\left(x_{k}, u_{k}, w_{k}\right)$ with respect to $x_{k}$

$$
A_{k}=\left[\begin{array}{ccccc}
0 & 0 & 0 & \frac{1}{m} & \frac{1}{m} \\
0 & 0 & 0 & \frac{R_{\omega}}{I_{\omega}} & 0 \\
0 & 0 & 0 & \frac{R_{\omega}}{I_{\omega}} & 0 \\
0 & 0 & 0 & 0 & 0 \\
0 & 0 & 0 & 0 & 0
\end{array}\right]
$$

$H_{k}$ is the Jacobian matrix of partial derivative of $h\left(x_{k}, v_{k}\right)$ with respect to $x_{k}$

$$
H_{k}=\left[\begin{array}{lllll}
0 & 0 & 0 & \frac{1}{m} & \frac{1}{m}
\end{array}\right]
$$

\section{Estimation of Friction Coefficient}

The slip slope $k_{s}$ is determined by the longitudinal tire force $F_{x}$, vertical tire force $F_{z}$ and longitudinal slip ratio $s_{X}$.

$$
\begin{aligned}
& \frac{F_{x}}{F_{z}}=k_{x} s_{x} \\
& F_{x}=F_{x f}+F_{x r}=k_{s} F_{z f} s_{x f}+k_{s} F_{z r} s_{x r} \\
& =k_{s}\left(F_{z f} s_{x f}+F_{z r} s_{x r}\right)
\end{aligned}
$$

According to the estimated tire longitudinal force and slip ratio, the slip slope can be estimated by the RLS method. The RLS method can be described by the following equation according to equation (9):

$$
y(t)=\varphi^{T}(t) \theta(t)+e(t)
$$

where $\theta(t)$ is the estimated parameters, which is slip slope in this research. $y(t)$ is the total longitudinal tire force and $e(t)$ is the error. $\varphi(t)$ is the regression vector, which is $F_{z f} s_{x f}+F_{z r} s_{x r}$.

The RLS algorithm has the procedure as followings at each time step [12]:

Step 1: Get the measurement value of the system output $y(t)$ and determine the regression vector $\varphi(t)$.

Step 2: According to the difference between system actual output at this time step and the system output predicted in the previous time step, determine the error $e(t)$

$$
e(t)=y(t)-\varphi^{T}(t) \theta(t-1)
$$

Step 3: The update gain vector $K(t)$ can be calculated as:

$$
K(t)=\frac{P(t-1) \varphi(t)}{\lambda+\varphi^{T}(t) P(t-1) \varphi(t)}
$$

And the covariance matrix $P(t)$ can be calculated by:

$$
P(t)=\frac{1}{\lambda}\left[P(t-1)-\frac{P(t-1) \varphi(t) \varphi^{T}(t) P(t-1)}{\lambda+\varphi^{T}(t) P(t-1) \varphi(t)}\right]
$$

Step 4: Update the estimated parameter

$$
\theta(t)=\theta(t-1)+K(t) e(t)
$$

$\lambda$ is called the forgetting factor, which is between (0.9, $1)$.

When the slip slope $k_{S}$ is estimated, based on the experimental data in the simulation, the tire-road friction coefficient can be estimated by the following equation:

$$
\mu=0.1 k_{s}+0.02
$$

\section{Simulation Results}

To validate the effectiveness of the proposed method, two different sets of simulations are conducted. In the first set of simulation, the vehicle is accelerating. In the second set of simulation, the vehicle is accelerating and then braking. The parameter values used in the simulations are given in Table 1.

TABLE I. SIMULATION PARAMETERS

\begin{tabular}{|l|l|}
\hline$m$ & $1298.9 \mathrm{~kg}$ \\
\hline$L_{f}$ & $1 \mathrm{~m}$ \\
\hline$L_{r}$ & $1.454 \mathrm{~m}$ \\
\hline$C_{S}$ & $50000 \mathrm{~N} / \mathrm{unit}^{2} \mathrm{slip}$ \\
\hline $\boldsymbol{I}_{\boldsymbol{z}}$ & $1627 \mathrm{kgm}^{2}$ \\
\hline$R_{W}$ & $0.35 \mathrm{~m}$ \\
\hline
\end{tabular}




\begin{tabular}{|l|l|}
\hline$I_{\omega}$ & $2.1 \mathrm{kgm}^{2}$ \\
\hline$\mu$ & 0.9 \\
\hline$\varepsilon_{r}$ & $0.015 \mathrm{~s} / \mathrm{m}$ \\
\hline$C_{\alpha}$ & $30000 \mathrm{~N} /$ unit slip \\
\hline$K$ (control gain in figure 2) & 100 \\
\hline
\end{tabular}

\section{A. The First Set of Simulation}

In this simulation, the traction torque is applied at the beginning as the following figure. The input traction torque is shown in Figure 2. The friction coefficient is set as 0.9 in the vehicle dynamics model.

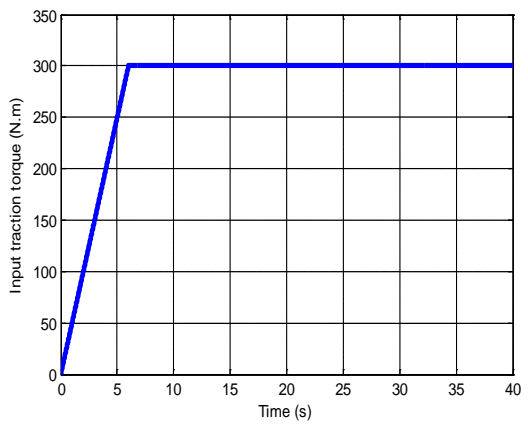

Figure 4. The vehicle input traction torque.

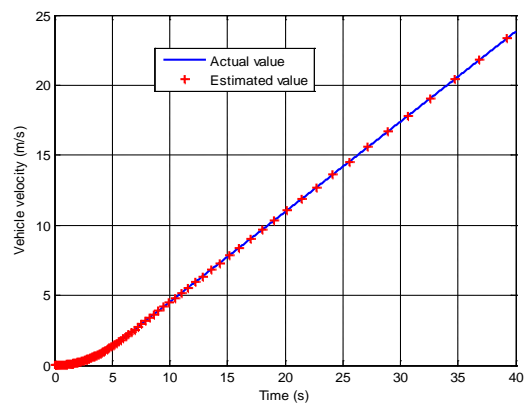

Figure 5. Compare the estimated vehicle absolute velocity with the actual value.

Figure 5 compares the actual vehicle velocity with the estimated vehicle velocity, which proves that the nonlinear filter in equation (4) can successfully estimate the vehicle absolute velocity.

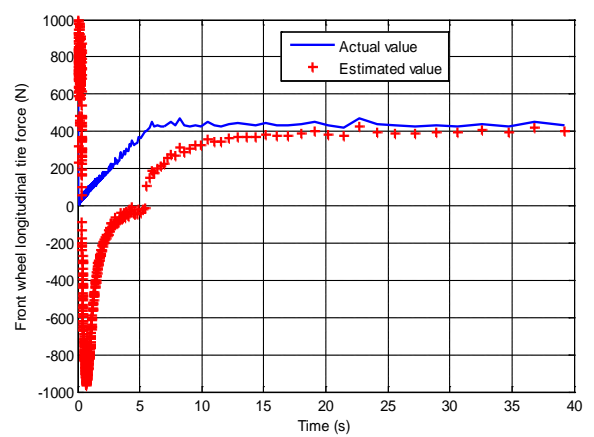

Figure 6. The estimated front tire longitudinal force.

Figure 6 shows the tire force estimation results of the applied EKF. The shape of the force curve in figure 6 is similar to the shape of the engine torque in Figure 4. This proves that the force estimation result of the EKF is accurate.

Figure 7 compares the actual friction coefficient and the estimated friction coefficient, which shows that the estimation result is acceptable because the estimated value is closing to the actual value. From $6 \mathrm{~s}$, the traction torque is applied on the vehicle and consequently the friction coefficient estimator can successfully find the value after 6 s.

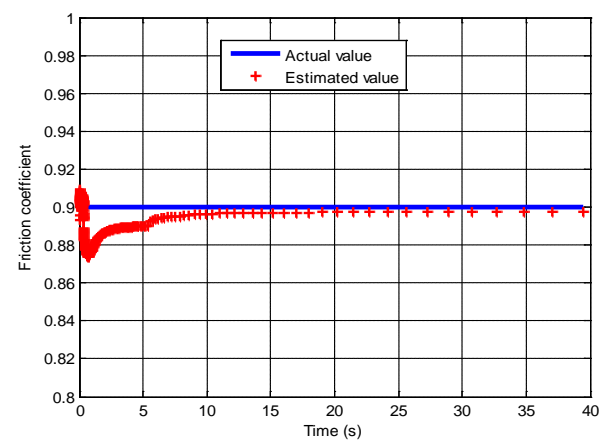

Figure 7. The estimated friction coefficient.

\section{B. The Second Set of Simulation}

In this simulation, the traction torque is applied at first. Then the brake torque is applied at 20s as shown in Figure 8. The friction coefficient is set as 0.9 in the vehicle dynamics model.

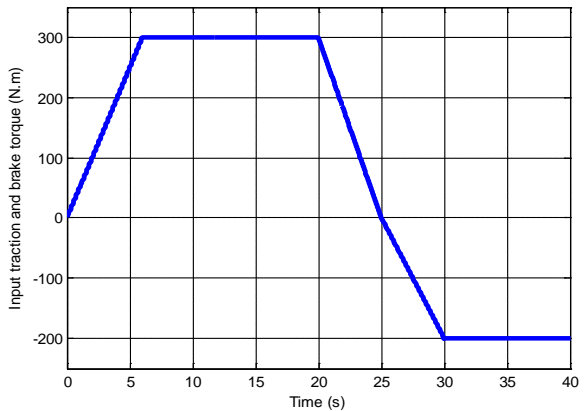

Figure 8 . The vehicle input traction and brake torque.

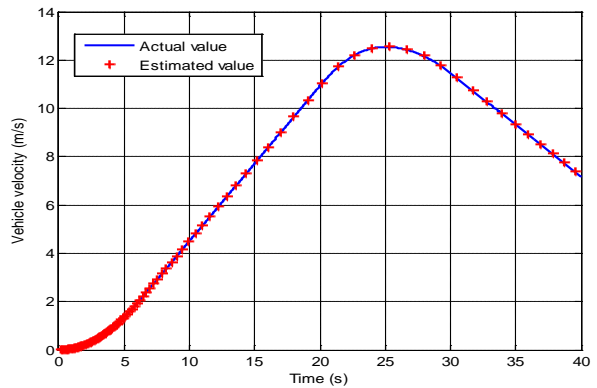

Figure 9. The vehicle velocity.

Figure 9 compares the actual vehicle velocity with the estimated vehicle velocity, which proves that the nonlinear filter in equation (4) can also successfully estimate the vehicle absolute velocity in this case. 
Figure 10 shows the tire force estimation results of the EKF. The shape of the force curve in figure 10 is similar to the shape of the engine torque in Figure 8 . This proves that the estimation results of the EKF is accurate.

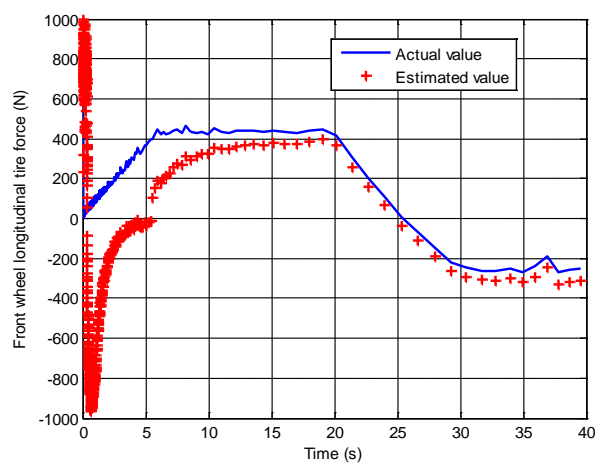

Figure 10. The estimated front tire longitudinal force.

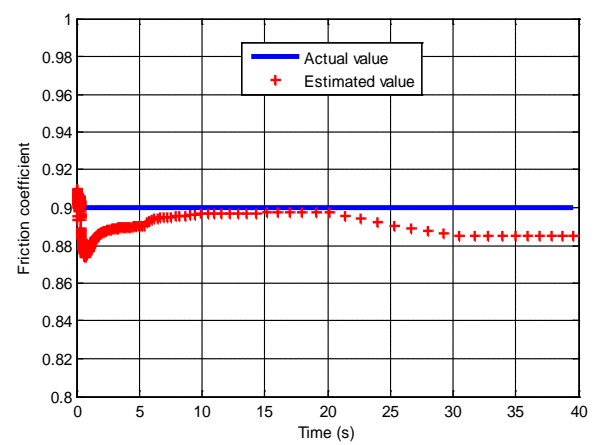

Figure 11. The estimated friction coefficient.

Figure 11 compares the actual friction coefficient and the estimated friction coefficient, which shows that the estimation result is also acceptable. However, from $20 \mathrm{~s}$, the vehicle is changing from the traction into brake and the estimated friction coefficient is decreasing, but the estimation result is still acceptable.

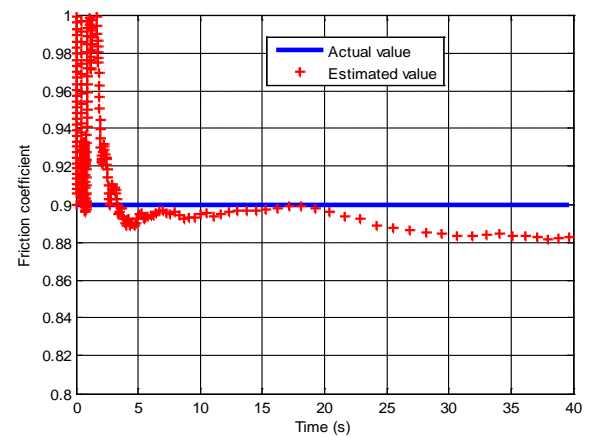

Figure 12. The estimated friction coefficient under random noise input.

In Figure 12, the random noise between $(-0.05,0.05)$ is applied on the measurement signal of longitudinal acceleration and the estimated friction coefficient is still acceptable, which verify the robustness of proposed estimator.

\section{CONCLUSION}

The slip slope method can only estimate the vehicle average friction coefficient. The individual tire force estimation method can estimate the friction coefficient of each tire but is not reliable. The EKF method in the literature needs to measure the tire force, which needs expensive sensors. In this paper, a novel cost effective tire-road friction coefficient estimation method is proposed here. This method only needs to measure the wheel angular velocity, the traction/brake torque and the longitudinal acceleration, which are all easy to be measured using available sensors installed in passenger vehicles. The vehicle absolute velocity can be estimated by the nonlinear filter without using the expensive GPS. In addition, the individual tire-road friction coefficient can be estimated by this method. Simulation examples are used to validate the effective of the proposed method. In the future, the real experiments will be conducted to verify the proposed estimator.

\section{REFERENCES}

[1] H. E. Tseng, D. Madau, B. Ashrafi, T. Brown, and D. Recker, "Technical challenges in the development of vehicle stability control system”, in Proc. IEEE Int.Conf.Control Applications, HI, Aug.1999, pp.1660-1666.W.-K. Chen, Linear Networks and Systems (Book style). Belmont, CA: Wadsworth, 1993, pp. 123-135.

[2] A. T. van Zanten, "Bosch ESP systems: 5 years of experience," in Proc. SAE Automotive Dynamics and Stability Conf., Troy. MI, May 2000, Paper No. 2000-01-1633.

[3] A. Nishio, K. Tozu, H. Yamaguchi, K. Asano, and Y. Amano, "Development of vehicle stability control system based on vehicle sideslip angle estimation,” SAE Paper, No. 2001-01-0137.

[4] L. Austin and D. Morrey, "Recent advances in antilock braking systems and traction control systems," Proc.Inst.Mech.Eng.D, vol.214, no.6, pp.625-638, 2000.

[5] K. Yi and J. Taeyoung, "Observer based estimation of tire-road friction for collision warning algorithm adaptation,” JSME Int.J.Series C. vol.41, no.1, pp.116-124, Mar.1998.

[6] G. Erdogan, L.Alexander, and R.Rajamani, "Friction coefficient measurement system for autonomous winter road maintenance", Vehicle System Dynamics, vol.47, no.4, pp.497-512, 2009.

[7] F. Gustaffson, 1997, "Slip-Based Tire-Road Friction Estimation," Automatica, vol.33, no.6, pp.1087-1099.

[8] S. Müller, M. Uchanski, and H. Karl, "Slip-Based Tire-Road Friction Estimation During Braking," Proceedings of 2001 ASME International Mechanical Engineering Congress and Exposition, New York, pp.213-220.

[9] J. Wang, L. Alexander, and R. Rajamani, "Friction estimation on high-way vehicles using longitudinal measurements”, ASME J. Dyn. Syst.,Meas. Control, vol.126, no.2, pp.265-275, 2004.

[10] L. R. Ray, "Nonlinear tire force estimation and road friction identification: Simulation and experiments,” Automatica, vol.33, pp. 1819-1833, 1997.

[11] J. Dakhlallah, S. Glaser, S. Mammar, and Y. Sebsadji, “Tire-Road Forces Estimation Using Extended Kalman Filter and Side slip Angle Evaluation”, 2008 American Control Conference, USA, 2008.

[12] R. Rajamani, G. Phanomchoeng, D. Piyabongkarn, and J. Y. Lew, "Algorithms for Real-Time Estimation of Individual Wheel Tire-Road Friction Coefficients”, IEEE/ASME Transactions on Mechatronics, vol.17, no.6, 2012.

[13] F. Jiang, and Z. Gao, “An Adaptive Nonlinear Filter Approach to the Vehicle Velocity Estimation for ABS”, Proceedings of the 2000 IEEE International Conference on Control Applications, USA, 2000.

[14] G. Welch, and G. Bishop, "An Introduction to the Kalman Filter”, September 17, 1997. 